\title{
A Case Report of Toxic Leukoencephalopathy induced by metronidazole in a woman with suture infection
}

\author{
Hoorvash Faraji Dana ${ }^{1}$, Lida Shojaei Arani ${ }^{1}$, Ali Faraji ${ }^{2}$, and Kiumars Bahmani ${ }^{3}$ \\ ${ }^{1}$ Shahid Beheshti University of Medical Sciences School of Medicine \\ ${ }^{2}$ Alborz University of Medical Sciences \\ ${ }^{3}$ Shahid Beheshti University of Medical Sciences
}

March 30, 2021

\begin{abstract}
Metronidazole may rarely cause encephalopathy and neuropathy. In this study we report a 30-year-old post-partum, ex-addicted female with leukoencephalopathy due to metronidazole.
\end{abstract}

\section{A Case Report of ToxicLeukoencephalopathy induced by metronidazole in a woman with suture infection}

Hoorvash Faraji Dana ${ }^{1}$, Lida Shojaei Arani ${ }^{1}$, Ali Faraji ${ }^{2}$, Kiumars Bahmani ${ }^{3 *}$

1. MD, Clinical Toxicology Fellowship, Department of Clinical Toxicology, Loghman-Hakim Hospital Poison Center, Faculty of Medicine, Shahid Beheshti University of Medical Sciences, Tehran, Iran

2. Medical Student, Student Research Committee, School of Medicine, Alborz University of Medical Sciences, Karaj, Alborz, Iran

3. PhD student of Toxicology, Department of Pharmacology and Toxicology, School of Pharmacy, Shahid Beheshti University of Medical Science, Tehran, Iran

Corresponding author email: Kiumarsbahmani@yahoo.com

\section{Key Clinical Message:}

Metronidazole may rarely cause encephalopathy and neuropathy. In this study we report a 30-year-old post-partum, ex-addicted female with leukoencephalopathy due to metronidazole.

\section{Introduction:}

Metronidazole is an antimicrobial agent used to treat various infections such as pseudomembranous colitis resulting from Clostridium difficile, amebiasis, giardiasis, trichomonas vaginitis and serious anaerobic bacterial infections ${ }^{1}$. Metronidazole may cause various neurological side effects including peripheral neuropathy, cerebellar dysfunction, visual impairment, vestibulotoxicity, cochleotoxicity, ataxic gait, dysarthria, seizures and encephalopathy ${ }^{2-4}$. In this study we report a case of metronidazole induced leukoencephalopathy.

\section{Case Presentation:}

A 30-year-old woman who had given birth two weeks ago was admitted to a regional hospital due to fever, palpitation, erythema, inflammation and secretion around the cesarean wound. Her vital signs were: T:37.3 BP:110/70 PR:89. Initial laboratory findings revealed R.B.C: $4.4 \times 106 / \mu \mathrm{L}$, Hb:11.6 g/dL, Hct: 36\%, W.B.C: $11.6 \times 103 / \mu \mathrm{L}$, Plt: $213 \times 103 / \mu \mathrm{L}$, Neutrophil: $73 \%$, Lymphocyte: $18 \%$, Monocyte: $5 \%$, Eosinophil: $5 \%$. So Primary diagnosis set for wound infection. Because of infection Amp Cefazolin (1 gr IV for 9 doses) 
and Amp Gentamicin (80 mg IM for 6 doses) and Amp Metronidazole (500 mg IV 7 doses) were ordered. After five days she complained of hearing loss so Gentamicin was stopped. She was also ex-addicted and taking methadone during abstain.

At day 9 of admission, she developed neural complications including nausea, vomiting, aphasia, loss of consciousness, hearing disturbances, extreme rigidity and gazed eyes so she was referred to Alborz hospital. There initial laboratory tests were as following: R.B.C: $4.7 \times 106 / \mu \mathrm{L}, \mathrm{Hb}: 13.5 \mathrm{~g} / \mathrm{dL}, \mathrm{Hct}: 41.3 \%$, Plt: $213 \times 103 / \mu \mathrm{L}$, W.B.C: $15.4 \times 103 / \mu \mathrm{L}$ (Neutrophil: $92 \%$, lymphocyte: $8 \%$ ), AST: $29 \mathrm{IU} / \mathrm{L}$, ALT: 18 IU/L, Sodium: $136 \mathrm{mEq} \backslash \mathrm{L}$, Potassium: $4.4 \mathrm{mEq} / \mathrm{L}, \mathrm{CRP}: 33, \mathrm{PH}: 7.307, \mathrm{PCO} 2:$ 26.9, HCO3: 13.2, PO2: 185.8, SO2: \%99.2, Urea 28, Cr: $1 \mathrm{mg} \backslash \mathrm{dL}$. There were evidences proving inflammation existence, So HBsAg, HCVAb, HIVab and blood culture was set which all were negative. Iso Electric Focusing for CSF \& Serum was done and OCB was negative. CSF culture, fluid examination and oligo clonal banding test didn't show any abnormality (Table1,2,3).

Brain MRI performed in axial T1, T2, FLAIR, DW and sagittal and coronal T2 sequences to examine brain functions in which diffuse white matter $\mathrm{T} 2$ increased signal changes were seen in periventricular regions on both sides and also in the corpus callosum. That showed restriction on DWI images. (figure 1). These findings are non-specific and could be due to inflammatory-infective causes as ADEM, HIV, PRES or vascular causes as CADASIL, so it was referred to molecular detection section for CSF pathogen panel. CSF viral pathogen panel showed negative for EBV, CMV, HSV1, HSV2, VZV, HHV 6, HHV7, Parvovirus B19, Human enterovirus.

At the entrance to Alborz hospital (9 days after first admission), she was hospitalized in ICU and took Methylprednisolone 1gr/day for 5 days, which was not effective, after that Plasmapheresis was done for 7 times by the amount of 2-2.5 liters with the substitution of FFP and Albumin which was again ineffective. She was ordered to 130 gr IVIG infused in divided doses in 5 days. After the patient was stable, she was ordered to MRI again in which Mild dilation of ventricle along with widening of lateral sulcus was noted. Cerebellar hemispheres vermis and dentate nucleus was visualized normally. Pathologic signal is not demonstrated in pons and medulla oblongata. Craniocervical junction appears normal. Extra or intra-axial collection in dura and meninges was not seen. signal void was seen normally in major arteries. Pathologic signal was not visualized in intracranial veins. There was not any space occupying lesion in basal cisterns. visualized parts of orbit had normal configuration. Macroadenoma was not seen in hypophysis. Stalk was normal. Suprasellar cistern was free. In DWI images, diffusion restriction and acute infarction was not seen Severe mucosal thickening was noted at ethmoid complex and also sphenoid sinus and less than severely at mastoid air cells (figure 2). At last, after 52 days she could talk and move voluntarily and ordered to take Depakine, Metoral, Plazil, Gemfibrozil, and Quetiapine.

\section{Discussion:}

Metronidazole can rarely cause central nervous system toxicity. Although Metronidazole-induced encephalopathy (MIE) is mostly reported from the United States and Korea, there have been few case reports worldwide including India, Japan, Australia, Canada, United Kingdom, Belgium, Chile, Germany, Israel, Netherlands, Nigeria, Taiwan, Tunisia, and Turkey. Average time taken to develop complications from treatment initiation is 15 days (range 1-90 days), and the average cumulative dose is $93.4 \mathrm{~g}$ (range, 0.25-1095g) ${ }^{5}$. MIE can be suspected in a patient presents cerebellar and brainstem deficits in close relation to metronidazole treatment ${ }^{6}$. T2/FLAIR hyperintense lesions in the dentate nuclei, are the best diagnostic finding. In some cases, lesions of the dentate nuclei were not present initially but revealed on re-imaging ${ }^{7,8}$. The majority of the cases present cerebellar dysfunction (dysarthria, ataxia, dysmetria, and nystagmus) followed by altered mental status and seizures ${ }^{5}$, Chorea, myoclonus ${ }^{9,10}$ and pure sensorineural hearing loss are also reported ${ }^{11}$. The patient has also taking methadone and there is reports of toxic leukoencephalopathy due to methadone ${ }^{12}$.

Toxic leukoencephalopathy should be considered in this patients and suspected drug should be discontinued.

\section{Conflict of interest :}


The authors declare there is no conflict of interest.

\section{Author Contribution:}

Dr. Hoorvash Faraji Dana was responsible for management of the patient, Dr. Lida Shojaei Arani was responsible for consultation and also patient management, Dr. Ali Faraji collected the data and drafting the case presentation section, Dr. Kiumars Bahmani drafting the manuscript and reviewed the literature.

\section{References:}

1. Roy U, Panwar A, Pandit A, Das SK, Joshi BJJoc, JCDR dr. Clinical and neuroradiological spectrum of metronidazole induced encephalopathy: our experience and the review of literature. 2016;10(6):OE01.

2. Bradley WG, Karlsson I, Rassol CJBmj. Metronidazole neuropathy. 1977;2(6087):610.

3. De Bleecker JL, Leroy BP, Meire VIJEn. Reversible visual deficit and corpus callosum lesions due to metronidazole toxicity. 2005;53(2):93-95.

4. Huang Y-T, Chen L-A, Cheng S-JJANT. Metronidazole-induced encephalopathy: case report and review literature. 2012;21(2):74-78.

5. Kuriyama A, Jackson JL, Doi A, Kamiya TJCn. Metronidazole-induced central nervous system toxicity: a systematic review. 2011;34(6):241-247.

6. Sørensen CG, Karlsson WK, Amin FM, Lindelof MJJon. Metronidazole-induced encephalopathy: a systematic review. 2020;267(1):1-13.

7. Eren F, Aldan MA, Dogan VB, Gül G, Selcuk HH, Soysal AJIs. A case with reversible neurotoxicity induced by metronidazole. 2017;70(11-12):429-432.

8. Singh R, Kaur R, Pokhariyal P, Aggarwal RJTIjor, imaging. Sequential MR imaging (with diffusionweighted imaging) changes in metronidazole-induced encephalopathy. 2017;27(2):129.

9. Foreid H, Coelho M, Ferreira J, Ferro JMJCR. Reversible iatrogenic subacute cerebellar syndrome, myoclonus and MRI findings following metronidazole administration. 2009;2009:bcr0520091841.

10. Galvez M, Brahm J, Miranda MJMD. Movement disorders as a manifestation of metronidazole-induced encephalopathy in a patient with chronic liver disease. 2009;24(12):1864-1866.

11. Jafari G, Hosseini SM, Akhondzadeh SJDJoPS. Sudden hearing loss subsequent to diarrhea: what is the missing link? 2014;22(1):15.

12. Haghighi-Morad M, Naseri Z, Jamshidi N, Hassanian-Moghaddam H, Zamani N, Ahmad-Molaei LJBMI. Methadone-induced encephalopathy: a case series and literature review. 2020;20(1):6.

Table 1: CSF Culture

\begin{tabular}{ll}
\hline Test & Result \\
Gram stain: & - \\
W.B.C & $0-1$ \\
Epith.Cells & $0-1$ \\
Bacteria & Not seen \\
Culture & No growth after $24 \mathrm{hrs}$. \\
& No growth after $48 \mathrm{hrs}$. \\
& No growth after $72 \mathrm{hrs}$. \\
Anaerobic culture & No growth \\
\hline
\end{tabular}

Table 2: C.S.F Fluid 


\begin{tabular}{llll}
\hline Test & Result & Unit & Reference Range \\
Appearance & Clear & & Clear \\
Color & Color less & & Clear Crystal color less \\
W.B.C & 0 & $\mathrm{~mm}^{3}$ & $<5 / \mu$ lit \\
R.B.C & 2 & $\%$ & $60-70 \%$ lymphocyte \\
Cell differential & - & & $30-35 \%$ monocyte \\
Glucose & 99 & $\mathrm{mg} / \mathrm{dL}$ & $>2 / 3$ blood glucose \\
Protein & 202 & $\mathrm{mg} / \mathrm{dL}$ & $<500$ \\
\hline
\end{tabular}

Table 3: Oligo Clonal Banding

\begin{tabular}{llll}
\hline Test & Result & Unit & Reference Range \\
Albumin Serum & $3.5 \mathrm{~g} / \mathrm{dL}$ & $\mathrm{g} / \mathrm{dL}$ & $3.5-5$ \\
CSF Albumin & $19 \mathrm{mg} / \mathrm{dL}$ & $\mathrm{mg} / \mathrm{dL}$ & $10-35$ \\
IgG & $1153 \mathrm{mg} / \mathrm{dL}$ & $\mathrm{mg} / \mathrm{dL}$ & $800-1700$ \\
IgG CSF & $\mathrm{mg} / \mathrm{dL}$ & Up to 80 \\
IgG index & & $3-0.7$ \\
Alb index & 0.6 & & $<9:$ intact BBB 9-12: \\
& 5.4 & & Slight impairment 12-20: \\
& & Moderate impairment \\
& & $30-100:$ Sever impairment \\
& & $>100$ : Complete \\
& & impairment \\
\hline
\end{tabular}

\section{Figure legends:}

Figure 1: brain MR in axial T1, T2, FLAIR, DW and sagittal and coronal T2 sequences. Increased signal changes in periventricular regions on both sides and also in the corpus callosum.

Figure 2: brain MRI without contrast. Mild dilation of ventricle along with widening of lateral sulcus, Severe mucosal thickening at ethmoid complex and also sphenoid sinus and less than severely at mastoid air cells. 

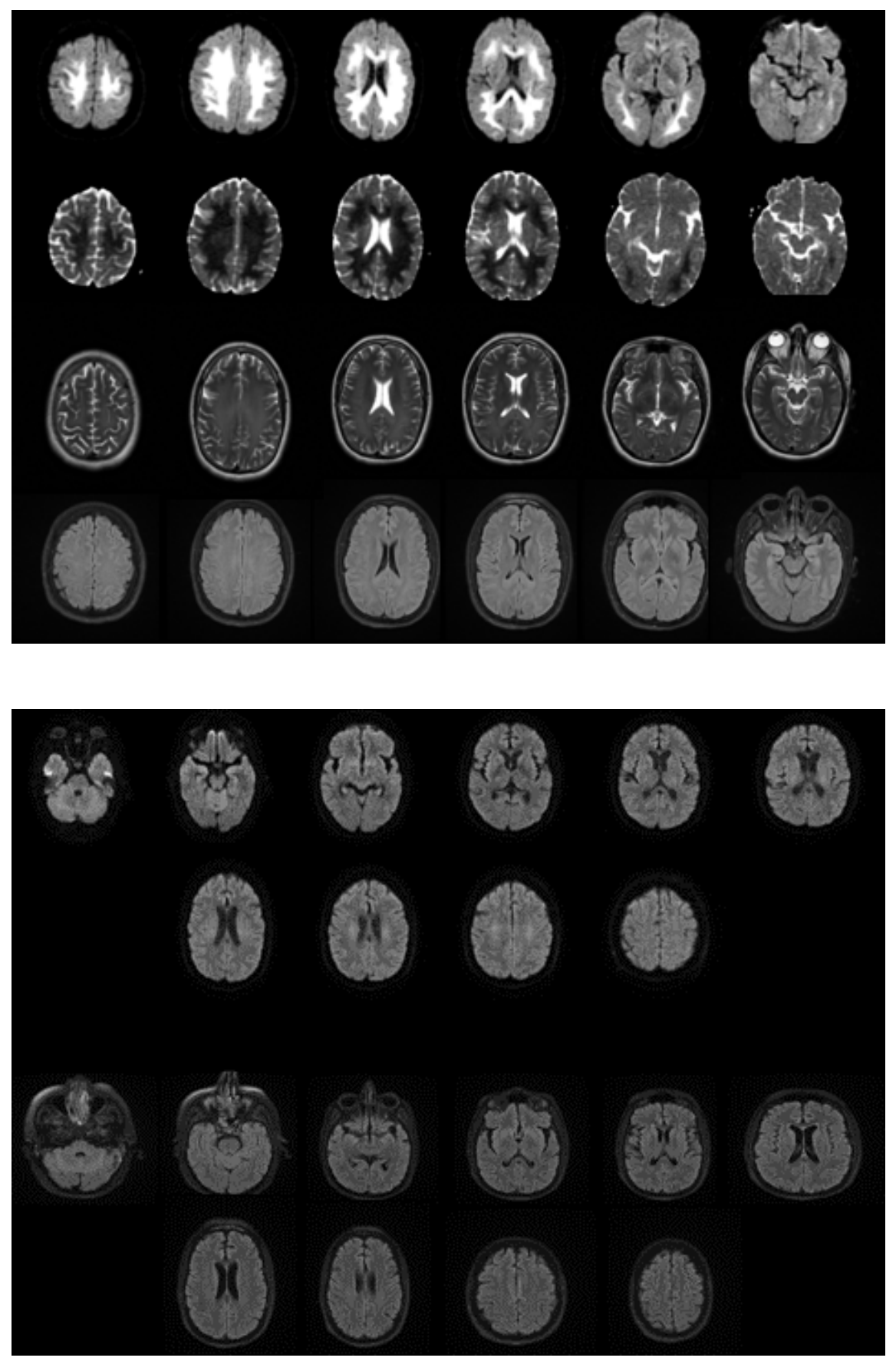